\title{
Motivação e emoções na aprendizagem da matemática no ensino básico
}

\section{Motivation and emotions in learning mathematics at elementary school}

\author{
Filipa Dinis, Ana Borgas Leal, Francisco Peixoto, Lourdes Mata \\ ISPA - Instituto Universitário
}

\begin{abstract}
Resumo
Pretendeu-se caraterizar a motivação e as emoções associadas à matemática, e analisar de que modo estas variam em função do desempenho nesta disciplina e do género dos alunos. Participaram no estudo 232 crianças portuguesas do terceiro ano do ensino básico, que responderam ao AEQ-ES e à MMS. Os resultados evidenciaram caraterísticas motivacionais positivas dos alunos face à matemática. Também a emoção positiva, prazer, surgiu mais associada à matemática do que as negativas. Constatou-se pouco efeito do género tanto na motivação como nas emoções. Relativamente ao desempenho, este surgiu significativamente associado às caraterísticas motivacionais e emoções vivenciadas pelos estudantes.
\end{abstract}

Palavras chave: motivação, emoções, aprendizagem, matemática

\begin{abstract}
This research aimed to characterize motivation and emotions related to mathematics, and to analyse the way in which these variables vary according to student's achievement and gender. Participants were 232 Portuguese students from the third grade of elementary school, completing the AEQ-ES and the MMS. Results showed that students presented positive motivational characteristics towards mathematics. The positive emotion, enjoyment, also appeared to be more related to mathematics than negative emotions. Gender revealed almost no effect either on motivation or emotions. Achievement emerged significantly associated to motivational characteristics and to emotions experienced by students.

Keywords: motivation, emotion, learning, mathematics
\end{abstract}

As competências no domínio da matemática têm sido vistas como capazes de auxiliar os alunos a tomar decisões e a fazer julgamentos fundamentados (OCDE, 2016), sendo apontadas como tendo um grande impacto na vida de um indivíduo, nomeadamente nas oportunidades profissionais a que se tem acesso (OCDE, 2014). Apesar da importância atribuída à disciplina da matemática, tem-se constatado que alguns alunos portugueses continuam a apresentar um desempenho inferior ao que seria expectável (Programme for International Student Assessment [PISA], 2012), não demonstrando melhorias significativas desde 2012 (OCDE, 2016). Deste modo, torna-se fundamental compreender e investigar diversos aspectos que possam estar relacionadas com os níveis de desempenho alcançados, de forma a encontrar melhores estratégias que possam alterar estes resultados.

Nos últimos anos, a investigação tem vindo a demonstrar que a motivação possui uma função chave na aprendizagem e no desempenho escolar em geral (Broussard \& Garrison, 2004), e na matemática em particular (Pintrich, Smith, García, \& McKeachie, 1993), tendo surgido várias teorias que procuram explicar quais os fatores que levam um indivíduo a envolver-se numa determinada tarefa. Uma dessas teorias é teoria de expetativa-valor (Eccles, 2006; Eccles et al., 1983, Wigfield \& Eccles, 2000), que defende que "as escolhas, persistência e desempenho do indivíduo podem ser explicadas pelas suas crenças relativas a quão bem-sucedido será na atividade e pela forma como valoriza a atividade" (Wigfield \& Eccles, 2000, p. 68). $\mathrm{Ou}$ seja, se uma atividade é importante para um indivíduo (valor) e ele acredita que terá um bom desempenho nesta atividade (expetativa), então terá um maior envolvimento nessa atividade do que numa outra que não considere importante, ou em que ache que não terá um bom desempenho (Roberts, 2007).

Eccles et al. (1983) definem expetativa de sucesso como a crença individual acerca de quão bem um indivíduo espera sair-se numa tarefa futura, refletindo esta crença a capacidade que o indivíduo tem de avaliar a sua competência numa determinada área. Os mesmos autores defendem uma definição abrangente de valor da tarefa, pois assumem que este é determinado por fatores como as características da tarefa, as necessidades gerais, os objetivos, os valores e as orientações motivacionais do indivíduo e, ainda, as memórias afetivas associadas a tarefas semelhantes realizadas no passado. Assim, preveem que os indivíduos se envolvam mais em tarefas que valorizam e que os valores dos indivíduos têm consequências ao nível da motivação e do comportamento (Eccles, 2006; Eccles et al., 1983). Neste sentido, consideram quatro componentes do valor da tarefa: valor de realização, valor intrínseco ou de interesse, valor de utilidade e custo. Para os autores o valor de realização está associado à importância pessoal de ter um bom desempenho numa dada tarefa em termos do esquema do self pois a tarefa fornece a oportunidade de demonstrar aspetos do self real ou ideal do indivíduo, tal como a masculinidade, feminilidade, e/ou competência em diversos domínios. 
$\mathrm{O}$ valor intrínseco ou de interesse prende-se com a satisfação que o indivíduo tem quando desempenha a atividade ou o interesse subjetivo que o indivíduo tem no tema. Por isso, quando o valor intrínseco é alto, o aluno tende a envolver-se mais na tarefa, a persistir mais e a continuar intrinsecamente motivado por ela (Ruiz, 2008).

O valor de utilidade é determinado pela forma como a tarefa se relaciona com objetivos atuais e futuros, tais como objetivos relacionados com a carreira. Uma tarefa pode ter um valor positivo para alguém, porque facilita objetivos futuros importantes, mesmo que a pessoa não esteja interessada na tarefa em si mesma.

Por último, o custo é identificado como um componente crítico do valor (Eccles, 2006; Eccles \& Wigfield, 2002). O custo é conceptualizado em termos dos aspetos negativos relativos ao envolvimento na tarefa, tais como a ansiedade relacionada com o desempenho e o medo, tanto do sucesso como do fracasso, assim como a quantidade de esforço necessária para ser bem-sucedido e as oportunidades perdidas que resultam de uma escolha em detrimento de outra (Eccles \& Wigfield, 2002).

O estudo do impacto do género é um tema recorrente na investigação em contexto escolar pelo que o estudo destes efeitos associados à aprendizagem da matemática não é exceção (Mata, Monteiro \& Peixoto, 2012). Eccles, Wigfield, Harold, e Blumenfeld (1993) reportam trabalhos envolvendo crianças tanto do ensino básico como do $2^{\circ}$ ciclo e secundário onde se constata que a perceção de competência e expetativa dos rapazes face à matemática é superior à das raparigas. As raparigas revelam uma maior preocupação em ter piores resultados a matemática e, apesar de nos primeiros anos do ensino básico elas até obterem frequentemente resultados superiores aos dos rapazes (Eccles et al., 1993), à medida que vão avançando na escolaridade, a baixa perceção de competência das raparigas face à matemática, associada a esta reação afetiva negativa medo de ter maus resultados - e ao aumento de dificuldade da disciplina, pode justificar um pior desempenho.

Complementarmente ao estudo da motivação, as emoções e o seu papel na aprendizagem e envolvimento dos alunos também têm sido considerados na investigação uma vez que, tal como referem Meyer e Turner (2006), os contextos educativos são desencadeadores de diversas emoções. Apesar disso, a investigação ao nível das emoções associadas à matemática tem-se focado maioritariamente na ansiedade, assim como no impacto que esta possui no desempenho dos alunos, e não tanto nas restantes emoções (Peixoto, Sanches, Mata \& Monteiro, 2017; Pekrun, 2006, 2009). Yurt (2015) constatou que os estudantes com expetativas mais elevadas face à matemática também reportavam maiores níveis de prazer face a atividades relacionadas com esta disciplina. Eram também estes alunos os que apresentam menos dificuldades e obtinham melhores resultados a matemática. Pekrun, Elliot e Maier (2006) também referem que existe uma associação entre as perceções de competência e as diferentes emoções experienciadas por um aluno. Lichtenfeld, Pekrun, Stupnisky, Reiss e Murayama (2012) sustentam que as emoções apresentam um impacto importante na motivação, na aprendizagem, no desempenho e mesmo no bem-estar dos alunos - por exemplo, o aborrecimento tem surgido associado a menores níveis de interesse e motivação, enquanto o prazer surge associado a níveis mais elevados de motivação intrínseca (Pekrun, Goetz, Frenzel, Barchfeld \& Perry, 2011).

Também nas emoções têm sido relatadas diferenças entre géneros, nomeadamente, os rapazes tendem a apresentar níveis mais elevados de prazer nas aulas de matemática (Pekrun et al., 2011); por outro lado, as raparigas apresentam níveis de ansiedade mais elevados durante tarefas que envolvam a matemática (Frenzel, Pekrun \& Goetz, 2007).

O presente estudo tem, assim, como objetivo caraterizar a motivação e as emoções associadas à matemática, e analisar de que modo estas variam em função do desempenho na disciplina, e do género, em alunos portugueses do $3^{\circ}$ ano do $1^{\circ}$ Ciclo do Ensino Básico.

\section{Método}

\section{Participantes}

Participaram neste estudo 232 estudantes (107 rapazes e 124 raparigas) a frequentar o terceiro ano do ensino básico público e privado, de diversas escolas da região de Lisboa.

\section{Instrumentos}

Motivação para a matemática. A motivação associada à matemática foi medida com recurso à Math Motivation Scale (MMS) (Peixoto, Mata, Radisic, Baucal, Laine \& Mononen, 2017), constituída por 23 itens, cotados numa escala de 4 pontos que varia entre 1 ("Muitas vezes") e 4 ("Nunca"). Os itens da MMS encontram-se divididos entre quatro dimensões: Interesse/Prazer, constituída por 6 itens (e.g. "Sinto-me bem quando faço atividades de matemática”); Utilidade, constituída por 6 itens (e.g. "Quando eu crescer será útil saber matemática"); Competência Percebida, constituída por 5 itens (e.g. "A matemática é fácil para mim”) e Custos, constituída por 6 itens (e.g. "Tenho de deixar de fazer muitas coisas para ter sucesso a matemática").

Emoções na matemática. Para aceder às emoções vivenciadas na sala de aula, em relação aos trabalhos e aos testes de matemática, recorreu-se ao Achievement Emotions Questionnaire - Elementary School (AEQ-ES) (Pekrun, Lichtenfeld, Killi, \& Reiss, 2007), constituída por 19 itens, divididos em três dimensões: Prazer, constituída por 8 itens (e.g., "Eu gosto da aula de matemática."); Ansiedade, constituída por 7 itens (e.g., "Fico tão nervoso durante um teste de matemática que não me consigo concentrar.") e Aborrecimento, constituída por 4 itens (e.g., "A aula de matemática aborrece-me."). Nesta escala, os alunos têm que se posicionar numa escala de resposta ancorada numa representação gráfica, que varia entre o 1 ("Nada") e o 5 ("Muito"). 
Foram realizadas análises à fiabilidade das dimensões de ambas as escalas, tendo estas revelado uma boa consistência interna (Tabela 1).

Desempenho. O desempenho foi medido através de um teste de matemática, constituído por 10 exercícios retirados da prova Trends in International Mathematics and Science Study (TIMSS).

\section{Procedimento}

Os dados utilizados neste estudo foram recolhidos diretamente nas escolas. A autorização de participação no estudo foi solicitada previamente aos pais por carta, tendo esta sido entregue ao professor responsável por cada turma. A participação dos alunos foi voluntária e salvaguardado o seu anonimato.

Os questionários foram administrados em sala de aula, sob supervisão de um membro da equipa de investigação. Sempre que se considerou necessário, alguns itens foram lidos em voz alta.

\section{Resultados}

Os resultados são apresentados de acordo com dois eixos principais - (a) estatísticas descritivas da motivação, das emoções e do desempenho dos participantes face à matemática, assim como as respectivas diferenças entre géneros, e (b) a análise de correlações entre as variáveis em estudo.

Tabela 1.

Fiabilidade das medidas utilizadas e estatísticas descritivas em função do género e para a totalidade dos participantes para as variáveis em estudo

\begin{tabular}{|c|c|c|c|c|c|c|c|c|c|}
\hline & & \multirow[t]{3}{*}{$\alpha$} & \multicolumn{5}{|c|}{ Género } & \multicolumn{2}{|c|}{ Global } \\
\hline & & & \multicolumn{2}{|c|}{ Masculino } & \multicolumn{2}{|c|}{ Feminino } & \multirow[b]{2}{*}{ Sig } & \multirow[b]{2}{*}{$M$} & \multirow[b]{2}{*}{$D P$} \\
\hline & & & $M$ & $D P$ & $M$ & $D P$ & & & \\
\hline \multirow{3}{*}{$\sum^{\infty}$} & $\begin{array}{l}\text { Interesse/ } \\
\text { Prazer }\end{array}$ & .90 & 3.53 & 0.71 & 3.37 & 0.70 & $\mathrm{~ns}$ & 3.44 & 0.71 \\
\hline & Utilidade & .82 & 3.71 & 0.53 & 3.79 & 0.40 & $\mathrm{~ns}$ & 3.75 & 0.47 \\
\hline & Custos & .78 & 1.91 & 0.73 & 2.04 & 0.74 & ns & 1.98 & 0.73 \\
\hline \multirow{5}{*}{ 焉 } & $\begin{array}{l}\text { Comp. } \\
\text { Perc. }\end{array}$ & .79 & 2.95 & 0.69 & 2.79 & 0.74 & $\mathrm{~ns}$ & 2.87 & 0.72 \\
\hline & Prazer & .92 & 4.06 & 0.99 & 3.78 & 1.05 & $*$ & 3.91 & 1.03 \\
\hline & Ansiedade & .91 & 2.11 & 1.05 & 2.35 & 1.07 & $\mathrm{~ns}$ & 2.24 & 1.07 \\
\hline & Abor. & .93 & 1.57 & 0.95 & 1.61 & 0.91 & $\mathrm{~ns}$ & 1.59 & 0.923 \\
\hline & Desemp. & - & 7.08 & 2.15 & 6.45 & 1.90 & $*$ & 6.75 & 2.04 \\
\hline
\end{tabular}

Na Tabela 1, são apresentados os valores das estatísticas descritivas (i.e., médias e desvios-padrão globais e por género das dimensões da MMS e da AEQES, assim como do desempenho), e os valores da fiabilidade (i.e., alfa de Cronbach). Os resultados da aplicação da MMS revelaram que os participantes se encontram, em média, motivados para a matemática, já que os valores obtidos se encontram acima do ponto médio da escala, nas dimensões Interesse/Prazer, Utilidade e Competência Percebida. No que se refere à dimensão Custos, verifica-se que estes são percecionados como baixos, o que corrobora a tendência para indicadores motivacionais bastante positivos, tanto no que se refere ao valor atribuído, como às expetativas de sucesso. Os resultados da análise de variância multivariada (MANOVA) indiciam a existência de efeitos do género na motivação para a matemática, Pillai's Trace $=.046, F(4,226)=2.73, p=.03$, os quais não são sustentados por diferenças significativas entre rapazes e raparigas, para nenhuma das dimensões da MMS. No entanto, verificam-se efeitos tendenciais nas dimensões Interesse/Prazer, $F(1,229)=2.805, p=.095$, e Competência Percebida $F(1,229)=2.80571, \quad p=.092$, dimensões em que os rapazes apresentam valores médios mais elevados.

No que respeita à análise das emoções face à matemática, os resultados da aplicação do AEQ-ES mostram que os participantes apresentam valores superiores ao ponto médio da escala na dimensão Prazer, mas valores inferiores ao ponto médio nas dimensões Ansiedade e Aborrecimento, demonstrando que as emoções associadas à matemática são positivas. A análise de variância multivariada (MANOVA) revelou efeitos do género nas emoções face à matemática, Pillai's Trace $=.043, \quad F(3,227)=3.42$, $p=.018$. As estatísticas univariadas revelam que esses efeitos se fazem sentir apenas na dimensão Prazer, $F(1,229)=4.424, p=.037$, com os rapazes a reportarem níveis de Prazer mais elevados que as raparigas.

No desempenho, os resultados dos participantes situam-se acima da média, havendo, contudo, uma diferença estatisticamente significativa entre géneros, sendo o desempenho dos rapazes mais elevado do que o das raparigas, $F(1,229)=5.652, p=.018$.

Tabela 2.

Correlações entre as dimensões da motivação, as emoções e o desempenho a matemática

\begin{tabular}{|c|c|c|c|c|c|c|c|c|c|}
\hline & & \multicolumn{4}{|c|}{ MMS } & \multicolumn{3}{|c|}{ AEQ } & \multirow{2}{*}{$\frac{\mathrm{D}}{8}$} \\
\hline & & 1 & 2 & 3 & 4 & 5 & 6 & 7 & \\
\hline 1 & $\begin{array}{l}\text { Interesse/ } \\
\text { Prazer }\end{array}$ & 1 & & & & & & & \\
\hline 2 & Utilidade & .54 & 1 & & & & & & \\
\hline$\sum 3$ & Custos & -.56 & -.32 & 1 & & & & & \\
\hline 4 & $\begin{array}{l}\text { Comp. } \\
\text { Perc. }\end{array}$ & .69 & .41 & -.55 & 1 & & & & \\
\hline$c^{5}$ & Prazer & .82 & .47 & -.48 & .67 & 1 & & & \\
\hline$\frac{1}{4} 6$ & Ansiedade & -.67 & -.37 & .45 & -.56 & -.62 & 1 & & \\
\hline 7 & Aborrec. & -.81 & -.54 & .52 & -.56 & -.77 & .66 & 1 & \\
\hline 8 & Desemp. & .36 & .25 & -.36 & .47 & .36 & -.30 & -.27 & 1 \\
\hline
\end{tabular}

Nota. MMS = Math Motivation Scale; AEQ = Achievement Emotions Questionnaire. Comp. Perc. = Competência percebida; Abor. = Aborrecimento; Desemp. = Desempenho. Todos os valores são significativos para $\mathrm{p}<.05$.

Seguidamente, realizou-se uma análise de correlações, tendo sido possível observar que as variáveis se encontram todas significativamente correlacionadas entre si (Tabela 2). Mais especificamente, verifica-se que valores mais elevados na dimensão Prazer (AEQ- 
ES) estão fortemente associados a valores mais elevados de motivação nas dimensões Interesse/Prazer e Competência Percebida (MMS) e que, por outro lado, o Aborrecimento (AEQ-ES) se encontra negativamente correlacionado com as dimensões Interesse/Prazer, Utilidade e Competência Percebida. No que respeita ao desempenho, as correlações encontradas são significativas, mas fracas, sendo que a mais forte é entre o Desempenho e a Competência Percebida (MMS).

\section{Discussão}

O presente estudo teve como objetivo caracterizar a motivação e as emoções associadas à aprendizagem da matemática, e analisar de que modo estas variam em função do desempenho nesta disciplina e do género, em estudantes do $3^{\circ}$ ano de escolaridade. Os resultados apresentados permitiram evidenciar que os participantes se encontram motivados para a disciplina de matemática e experienciam maioritariamente emoções positivas (i.e., Prazer) face à matemática, ao invés do que ocorre com as emoções negativas (i.e., Ansiedade, Aborrecimento).

No que se refere aos efeitos do género na motivação para a matemática, apesar das diferenças encontradas em termos globais, não foram evidenciadas diferenças nas dimensões consideradas. No entanto, os efeitos marginais encontrados nas dimensões Interesse/Prazer e Competência Percebida vão no sentido esperado, com os rapazes a apresentarem valores mais elevados do que as raparigas (e.g., Eccles et al., 1993). Ainda em relação à motivação, e apesar de na dimensão de Custo os resultados também não revelarem diferenças entre géneros, para o item "Fico aflito se não sou capaz de fazer os exercícios de matemática" as raparigas apresentam um valor significativamente superior aos dos rapazes. Este medo de errar, associado a uma Competência Percebida mais baixa pode justificar o desempenho inferior por parte das raparigas observado neste estudo, tal como sugerido por Eccles et al. (1993) e que é também consistente com o relatório da OCDE (2016), em que os rapazes apresentam um desempenho superior ao das raparigas.

Já no que diz respeito às emoções experienciadas, encontraram-se diferenças estatisticamente significativas entre géneros na dimensão Prazer, a favor dos rapazes, o que é consistente com os resultados reportados por Pekrun et al. (2011).

O presente estudo pretendia, ainda, compreender como é que as variáveis em estudo se relacionam entre si. Nesta medida, observou-se que a dimensão Prazer (AEQ-ES) se encontra fortemente correlacionada com as dimensões Interesse/Prazer (MMS), o que poderá ser explicado pelo facto de um aluno sentir mais prazer ao realizar uma tarefa que seja interessante; surge também moderadamente correlacionada com a Competência Percebida (MMS), o que é concordante com o encontrado por Yurt (2015), que refere que alunos com expectativas mais elevadas face à matemática são também aqueles que reportam maiores níveis de prazer. É ainda de referir que, de acordo com Pekrun et al. (2011), seria de esperar que emoções como o prazer surgissem positivamente associadas à motivação, o que é consistente com os resultados encontrados neste estudo.

Adicionalmente, verificou-se que o Aborrecimento (AEQ-ES) se correlaciona forte e negativamente com o Interesse/Prazer (MMS), e que a Ansiedade (AEQ-ES) também se relaciona com o Interesse/Prazer de modo negativo, embora apenas moderadamente. Pekrun (2006, 2009) postulou que emoções negativas poderiam interferir com a motivação, na medida em que poderiam reduzir o prazer retirado de uma tarefa (já que emoções negativas e positivas seriam incompatíveis), ou na medida em que um aluno passaria a evitar envolver-se em tarefas que suscitassem emoções desagradáveis deste modo, faz sentido que emoções como o Aborrecimento e a Ansiedade surjam negativamente associadas ao Interesse/Prazer.

$\mathrm{O}$ Aborrecimento relaciona-se ainda forte $\mathrm{e}$ negativamente com o Prazer, o que é consistente com o preconizado pela teoria de expetativa-valor (Pekrun, 2006, 2009) e com os resultados encontrados no estudo de Pekrun et al. (2011), no qual as emoções positivas se encontravam negativamente correlacionadas com as emoções positivas. Foi também possível observar que o Aborrecimento se correlaciona negativamente, embora de forma moderada, com valor pela Utilidade. Esta relação pode ser explicada tendo em conta que, caso os alunos não percecionem uma tarefa como sendo útil, é mais provável que demonstrem algum aborrecimento face à mesma. Encontrou-se igualmente uma relação negativa com a Competência Percebida, o que também poderá ser explicado, se considerarmos que os alunos se poderão sentir mais aborrecidos ao envolver-se numa tarefa na qual não pensam poder ser bem-sucedidos.

No que respeita ao desempenho, as correlações encontradas são fracas, indiciando que poderão existir outras variáveis e relações mais complexas (Peixoto et al, 2017). A relação mais forte foi entre o Desempenho e a Competência Percebida (MMS), o que também é justificável, já que, quanto mais competência o aluno considera ter, maior será o seu envolvimento na tarefa, o que levará a níveis mais elevados de desempenho.

Podemos então concluir que os resultados deste trabalho enfatizam a necessidade e importância de analisar as componentes afetivas associadas à aprendizagem, de modo a poder ter uma visão mais completa e integrada dos processos de aprendizagem.

\section{Referências}

Broussard, S., \& Garrison, M. E. (2004). The relationship between classroom motivation and academic achievement in elementary-school-aged children. Family and Consumer Sciences Research Journal, 33(2), 106-120. doi: 10.1177/1077727X04269573

Eccles, J. (2006). A motivational perspective on school achievement. Taking responsibility for learning, teaching, and supporting. In R. Sternberg \& R. Subotnik (Eds). Optimizing student success in school with the other three Rs - Reasoning, Resilience, and 
responsibility (pp. 199-224). Connecticut: IAP Information Age Publishing.

Eccles, J. S., Adler, T. F., Futterman, R., Goff, S. B., Kaczala, C. M., Meece, J., \& Midgley, C. (1983). Expectancies, values and academic behaviors. In J. T. Spence (Ed.), Achievement and achievement motives (pp. 75-146). San Francisco: Freeman

Eccles, J., Wigfield, A., Harold, R. D. \& Blumenfeld, P. (1993). Age and Gender Differences in Children's Self- and Task Perceptions during Elementary School. Child Development, 64 (3), 830-847. doi:10.1111/j.1467-8624.1993.tb02946.x

Eccles, J. S., \& Wigfield, A. (2002). Motivational beliefs, values, and goals. Annual Review of Psychology, 53, 109-132. doi: 10.1146/annurev.psych.53.100901.135153

Frenzel, A. C., Pekrun, R., \& Goetz, T. (2007). Girls and mathematics - a "hopeless" issue? A controlvalue approach to gender differences in emotions towards mathematics. European Journal of Psychology of Education 22(4), 497-514. doi:10.1007/BF03173468.

Lichtenfeld, S., Pekrun, R., Stupnisky, R., Reiss, K., \& Murayama, K. (2012). Measuring students' emotions in the early years: the Achievement Emotions Questionnaire-Elementary School (AEQ-ES). Learning and Individual Differences, 22(2), 190-201. doi:10.1016/j.lindif.2011.04.009.

Mata, L., Monteiro, V., \& Peixoto, F. (2012). Attitudes towards mathematics: Effects of individual motivational, and social support factors. Child Development Research. 2012, 1-10. doi: 10.1155/2012/876028

Meyer, D., \& Turner, J. (2006). Re-conceptualizing emotion and motivation to learn in classroom contexts. Educational Psychology Review. 18(4) 377390. doi:10.1007/s10648-006-9032-1

OCDE (2014). PISA 2012 results: What students know and can do - Student performance in mathematics, reading and science (Vol. 1).

OCDE (2016). PISA 2015 Results (Volume I): Excellence and Equity in Education, OECD Publishing, Paris. doi: 10.1787/9789264266490-en

Peixoto, F., Mata, L., Radisic, J., Baucal, A., Laine, A. T., \& Mononen, R.M. (2017). Validation of the Math Motivation Scale - Elementary School. Manuscript in preparation.

Peixoto, F., Sanches, C., Mata, L., \& Monteiro, V. (2017). "How do you feel about math?": Relationships between competence and value appraisals, achievement emotions and academic achievement. European Journal of Psychology of Education, 32, 385-405. doi: 10.1007/s10212-0160299-4

Pekrun, R. (2006). The Control-Value Theory of Achievement Emotions: Assumptions, Corollaries, and Implications for Educational Research and Practice. Educational Psychology Review, 18, 315341. doi: 10.1007/s10648-006-9029-9

Pekrun, R. (2009). Global and local perspectives on human affect: Implications of the controlvalue theory of achievement emotions. In M. Wosnitza, S. A.
Karabenick, A. Efklides, \& P. Nenninger (Eds.), Contemporary motivation research: From global to local perspectives (pp. 97-115). Cambridge, MA: Hogrefe.

Pekrun, R., Elliot, A. J., \& Maier, M. A. (2006). Achievement goals and discrete achievement emotions: A theoretical model and prospective test. Journal of Educational Psychology, 98(3), 583-597.

doi: 10.1037/0022-0663.98.3.583

Pekrun, R., Goetz, T., Frenzel, A. C., Barchfeld, P., \& Perry, R. P. (2011). Measuring emotions in students' learning and performance: The Achievement Emotions Questionnaire (AEQ). Contemporary Educational Psychology, 36, 36-48. doi: 10.1016/j.cedpsych.2010.10.002

Pekrun, R., Lichtenfeld, S., Killi, U., \& Reiss, K. (2007). Achievement Emotions Questionnaire Elementary School (AEQ-Elementary School) User's Manual. Munich, Germany: University of Munich, Department of Psychology.

Pintrich, P. R., Smith, D. A., García, T., \& McKeachie, W. J. (1993). Reliability and predictive validity of the Motivated Strategies for Learning Questionnaire (MSLQ). Educational and psychological measurement, 53(3), 801-813. doi: $10.1177 / 0013164493053003024$

Programme for International Student Assessment [PISA]. (2012). Portugal - Primeiros resultados. Recuperado de http://www.dgeec.mec.pt

Roberts, J. M. (2007). The expectancy-value theory of achievement motivation and the enumeration of possible selves: evidence for outcomes during emerging adulthood. Masters Theses and Doctoral Dissertations, 59

Ruiz, V. M. (2008). Valor de tarefas de aprendizagem para universitários de cursos noturnos. Revista Semestral da Associação Brasileira de Psicologia Escolar e Educacional, 12 (2), 451-460

Wigfield, A., \& Eccles, J. (2000). Expectancy-value theory of achievement motivation. Contemporary Educational Psychology, 25(1), 68-81. doi: 10.1006/ceps.1999.1015

Yurt, E. (2015). Understanding middle school students' motivation in math class: The expectancy-value model perspective. International Journal of Education in Mathematics, Science and Technology, 3(4), 288297. doi: 10.18404/ijemst.26938

\section{Agradecimentos}

Os autores agradecem às escolas que amavelmente permitiram a recolha dos dados e a todas as crianças que participaram no estudo. 